\title{
Evaluación del servicio ecosistémico de regulación hídrica ante escenarios de conservación de vegetación nativa y expansión de plantaciones forestales en el centro-sur de Chile
}

\author{
Assessment of the ecosystem service of water regulation under scenarios \\ of conservation of native vegetation and expansion of forest plantations \\ in south-central Chile
}

\author{
Cristobal Jullian a*, Laura Nahuelhual ${ }^{\text {b,c }}$, Bruno Mazzorana ${ }^{\text {, }}$, Mauricio Aguayo e,f \\ * Autor de correspondencia: a Universidad Austral de Chile, Facultad de Ciencias Forestales y Recursos Naturales, \\ Programa de Magister en Ciencias mención bosque y medio ambiente, Valdivia, Chile, cristobal.jullian@gmail.com \\ ${ }^{\mathrm{b}}$ Universidad Austral de Chile, Facultad de Ciencias Agrarias, Instituto Economía Agraria, 2do piso, \\ Casilla 567, Valdivia, Chile, tel.: 56-63-2221237. \\ ${ }^{\mathrm{c}}$ Centro de Investigación en Dinámica de Ecosistemas Marinos de Altas Latitudes (IDEAL), \\ Universidad Austral de Chile, Valdivia, Chile, tel.: 56-63-232567. \\ ${ }^{d}$ Universidad Austral de Chile, Instituto de Ciencias de la Tierra, Valdivia, Chile. \\ ${ }^{\mathrm{e}}$ Centro de Ciencias Ambientales EULA-Chile, Universidad de Concepción, Concepción, Chile. \\ ${ }^{\mathrm{f}}$ Universidad de Concepción, Instituto de Ecología y Biodiversidad (IEB), Concepción, Chile.
}

\section{SUMMARY}

Land use change (LUC) is one of the most conditioning biophysical factors in the hydric regulation although the magnitude of its effects has been little studied. The objective was to analyze the changes in the magnitude of the ecosystem service of water regulation under future scenarios of LUC at watershed scale, taking as case study the municipality of Panguipulli (Los Ríos Region, Southern Chile). The methodology was based on the Curve Number method applied through the ECOSER mapping protocol, which estimates the ability to regulate rainfall considering the type of vegetation and the physical characteristics of the soil. Modeling results showed that under a scenario A of increased old-growth forest cover (64 \%), watersheds increase their capacity to regulate between $0.4 \%$ and $7.1 \%$, while in a scenario of increased industrial forest ( $88 \%$ ), watersheds reduced their capacity to regulate between $0.3 \%$ and $0.9 \%$, considering a single precipitation event in 24 hours. The watersheds with the largest increase in regulation capacity $\left(\overline{\mathrm{X}}=11.8 \mathrm{~m}^{3}\right.$ ha-1) had a low initial area of native forest $(\overline{\mathrm{X}}=15 \%)$, a large area of soil type $\mathrm{A}$ and $\mathrm{B}(40 \%)$ and a smaller area of soil types $\mathrm{C}$ and $\mathrm{D}(21 \%)$. The basins with the largest reduction in regulation capacity $\left(\bar{X}=4.4 \mathrm{~m}^{3} \mathrm{ha}^{-1}\right)$ exhibited a low area of plantations $(\bar{X}=5 \%)$, a low area of soil type A and B (32\%) and higher area of soil type C and D (45\%). This information supports the need to focus land use planning efforts on watersheds most exposed to the expansion of plantations.

Key words: socio-ecological systems, eco-hydrology, geographic information system (GIS).

\section{RESUMEN}

El cambio de uso de suelo es uno de los factores biofísicos más condicionantes en la regulación hídrica cuyos efectos siguen siendo poco estudiados. El objetivo fue analizar cambios del servicio ecosistémico de regulación hídrica bajo escenarios de cambio de uso de suelo a escala de cuencas, en la comuna de Panguipulli, sur de Chile. La metodología se basó en el método Curva Número aplicado a través del protocolo ECOSER, el cual estima la capacidad de regulación hídrica desde las precipitaciones, considerando el tipo de vegetación y suelo. La modelación mostró que ante un escenario A de aumento de bosque nativo adulto (64 \%), las cuencas incrementan su capacidad de regular entre un $0,4 \%$ y un $7,1 \%$, mientras que ante un escenario de aumento de plantaciones forestales adultas $(88 \%)$, las cuencas mermaron su capacidad de regular entre un $0,3 \%$ y un $0,9 \%$, dependiendo de la cuenca. Las cuencas con mayor aumento de la capacidad de regulación en el escenario $\mathrm{A}\left(\overline{\mathrm{X}}=11,8 \mathrm{~m}^{3} \mathrm{ha}^{-1}\right)$ poseían baja superficie de bosque nativo $(\overline{\mathrm{X}}=15,4 \%)$, alta superficie de suelos tipo A y B (40 \%) y menor área del suelo tipo C y D $(21 \%)$. Las cuencas donde más se redujo la capacidad de regulación en el escenario $B\left(\bar{X}=4,4 \mathrm{~m}^{3} \mathrm{ha}^{-1}\right)$ fueron cuencas con baja superficie inicial de plantación $(\bar{X}=5,1 \%)$ y baja superficie de suelos tipo A y B (32 \%) y mayor del tipo C y D (45 \%). Esta información avala la necesidad de planificación de uso del suelo en cuencas.

Palabras clave: sistemas socio-ecológicos, eco-hidrología, sistemas de información geográficos (SIG). 


\section{INTRODUCCIÓN}

La influencia de los sistemas naturales en el balance y regulación de flujos hídricos en la superficie terrestre depende de los componentes que permiten almacenar agua en napas subterráneas, los cuales a su vez están influenciados en gran parte por la vegetación y propiedades físicas del suelo (Chen et al. 2010).

El servicio ecosistémico de regulación hídrica se refiere a la proporción de las precipitaciones que pueden ser interceptadas e infiltradas en napas subterráneas, contribuyendo al constante flujo de agua como escorrentía superficial. El proceso de regulación hídrica comienza con la intercepción de la precipitación incidente por el dosel de la vegetación, donde una gran proporción se pierde por evaporación. El agua residual de esta etapa, fluye a través del escurrimiento fustal, acoplándose a la precipitación directa la cual no es interceptada y llega directamente al suelo, donde una proporción fluye como escurrimiento superficial, otra se infiltra y se almacena en el suelo, quedando disponible para las plantas y el resto se acumula en napas subterráneas. Por ende, la regulación y almacenamiento de agua dependerá de estos procesos circunscritos al balance hídrico, donde el tipo de vegetación (v.g., tasas de evapotranspiración) y las características físicas del suelo (v.g., infiltración) condicionan la capacidad de regulación (Grizzetti et al. 2016).

Los acuíferos aportan agua como escorrentía sobretodo en periodos en que las precipitaciones disminuyen, mientras que su recarga dependerá de la capacidad de capturar y regular las lluvias. Sin embargo, el continuo aporte de agua por parte de los acuíferos, agota las fuentes o reservorios sin una recarga constante, hasta alcanzar un punto en que la capacidad de proveer agua comienza a ser limitada. Aunque la generación de escorrentía ante eventos de precipitación breves contribuye a la provisión de agua, la mayor parte de dicha provisión está estrechamente vinculada a los acuíferos y su capacidad de recarga y no a la respuesta hidrológica en períodos acotados de tiempo, por lo cual, la provisión hídrica queda condicionada por la capacidad de regulación y disponibilidad en acuíferos.

La evaluación del servicio ecosistémico de regulación hídrica requiere la modelación del sistema hidrológicoterrestre, el cual está sujeto a cambios permanentes como resultado de la evolución constante en el uso de la tierra (Li et al. 2007). Por ende, comprender la evolución pasada y las tendencias futuras en el cambio de uso de suelo es clave en el estudio de la dinámica de los procesos hidrológicos y los servicios ecosistémicos que de ellos se derivan. Sin embargo, todavía hay una comprensión limitada de cómo las alteraciones del uso de la tierra impactan los flujos de servicios ecosistémicos. Estudios recientes demuestran el potencial de los enfoques de modelización integrada para evaluar el impacto del cambio de uso de suelo en los servicios ecosistémicos hídricos (López-Moreno et al. 2014).
La región templada del sur de Chile, donde se lleva a cabo el presente estudio, exhibe una de las mayores tasas de deforestación (5,4 \% al año) en América Latina (Heilmayr et al. 2016) entre el período 2010-2015. Esto contrasta radicalmente con los datos presentados por la FAO donde Chile declara un aumento de hasta un $10 \%$ en la superficie boscosa entre 2010 y 2015 (FAO 2015), aporte que han hecho en su gran mayoría las plantaciones forestales. Durante el último decenio, el establecimiento de plantaciones forestales (v.g., Pinus radiata. D. Don, Eucalyptus nitens $\mathrm{H}$. Deane et Maiden), que condujo a la sustitución de la cobertura forestal nativa por plantaciones exóticas y forestación en tierras agrícolas despejadas, causando un cambio significativo en el paisaje y en los servicios ecosistémicos (Nahuelhual et al. 2012). El crecimiento de especies exóticas tiene consecuencias ecohidrológicas inciertas y los impactos ecológicos a su vez generan consecuencias poco claras y estudiadas (Heilmayr et al. 2016). Varios autores han concluido que las consecuencias de las plantaciones exóticas de rápido crecimiento son: (i) la disminución de la escorrentía en cuerpos de agua debido a la mayor evapotranspiración (Huber et al. 2008), (ii) cambios en las propiedades hidrológicas del suelo, como las tasas de infiltración (Oyarzún et al. 2011) y la hidrofobicidad del suelo (Ferreira et al. 2000), todo lo cual contribuye potencialmente a una menor capacidad de regulación hídrica.

El sur de Chile ofrece un escenario propicio para los estudios que sustentan la comprensión de las complejas interacciones entre cambio de uso de suelo y la provisión de servicios ecosistémicos en los países en desarrollo, fuera de los trópicos, debido a que aún mantiene gran parte de su superficie cubierta por bosque nativo, además, de un claro aumento de plantaciones forestales en él desde el año 1998 al 2013 (CONAF 1998, 2013), en paralelo a dinámicas persistentes de degradación del bosque nativo y su abandono.

Se proponen como hipótesis las siguientes: i) la regulación hídrica es afectada negativamente en cuencas hidrográficas donde las trayectorias de cambio de uso de suelo ocurren desde coberturas de matorral y matorral arborescente hacia coberturas de plantación; ii) la regulación hídrica es afectada positivamente en cuencas hidrográficas donde las trayectorias de cambio de uso de suelo ocurren desde coberturas como plantación, cultivo y pradera hacia coberturas de bosque nativo.

El objetivo de este trabajo es analizar los cambios en la magnitud y distribución espacial del servicio ecosistémico de regulación hídrica bajo escenarios futuros de cambio de uso de suelo para comprender el efecto en la hidrología de una cuenca de plantaciones forestales exóticas y el rol eco-hidrológico del bosque nativo en la capacidad de regulación hídrica, considerando el análisis a escala de cuencas hidrográficas y tomando como caso de estudio la comuna de Panguipulli, región de Los Ríos, sur de Chile $\left(39^{\circ} \mathrm{S}\right)$. 


\section{MÉTODOS}

Este estudio se llevó a cabo en la comuna de Panguipu$11 \mathrm{i}\left(39^{\circ} 38^{\prime} 0^{\prime \prime} \mathrm{S}, 72^{\circ} 20^{\prime} 0^{\prime \prime} \mathrm{O}\right)$, ubicada en la región de Los Ríos, Chile (figura 1).

El clima del área se define como templado lluvioso con influencia mediterránea, predominando los vientos del norte en invierno y oeste/sur en verano (METEOCHILE 2001). La pluviometría anual registrada en el año 2016 en el área alcanzó valores desde 1.395 y 2.524 mm (Dirección General de Aguas, DGA 2016). La topografía de la comuna se caracteriza por ser altamente accidentada, dominado por montañas, quebradas abruptas asociadas a fenómenos de erosión glacial. Desde los 600 hasta los $1.400 \mathrm{~m}$ s.n.m., existe una dominancia de suelos derivados de tefras, moderadamente profundos, con clase textural que varía desde franco arenosa gruesa hasta franco limosa y su permeabilidad es de moderada a rápida con drenaje bueno a excesivo (Luzio et al. 2009). La vegetación nativa está representada por las regiones vegetales del tipo bosques laurifolio valdiviano, bosque caducifolio andino y bosque andino patagónico (sub-región de las cordilleras patagónicas) (Gajardo, 1994).

Pasos metodológicos. La metodología se basó en la aplicación del protocolo ECOSER de mapeo de vulnerabilidad socioecológica frente al cambio de uso de suelo (Laterra 2015). En su Módulo 1, ECOSER permite mapear servicios ecosistémicos como producto de la interacción de funciones ecológicas y atributos del paisaje. Específicamente, el mapeo del servicio ecosistémico de regulación hídrica dentro de ECOSER se basa en el método Curva Número (CN en adelante) desarrollado en 1969 por el Servicio de Conservación de Suelos (SCS por sus siglas en inglés) perteneciente al Departamento de Agricultura de los Estados Unidos (Ahmad et al. 2015). El valor CN es un índice empírico cuyo objetivo es estimar la proporción de la precipitación que será retenida, evapotranspirada y la que se convertirá en escorrentía superficial, condicionada por el vínculo entre el suelo y la vegetación. Así, este trabajo utilizó la función ecosistémica "retención de exceso de precipitaciones por cobertura vegetal" propuesta por ECOSER, como indicador del servicio ecosistémico de regulación hídrica.

Para llevar a cabo el mapeo, se recopilaron bases de datos espaciales en formato raster y shapefile estandarizados (pixeles de 30x30m) los que se llevaron a una base de datos para su posterior análisis a través del software Arcgis 10.1 y la extensión creada para Acrtoolbox "ECOSER 2.0". Los insumos geoespaciales e información que requirió ECOSER fueron: i) valores $\mathrm{CN}$, ii) precipitación y iii) número de tormentas por año (para este caso se tomó valor " 1 " con el fin de evaluar la capacidad solo ante un evento en particular).

La metodología se sintetiza en el siguiente diagrama (figura 2) y cada paso es explicado en la siguiente sección.

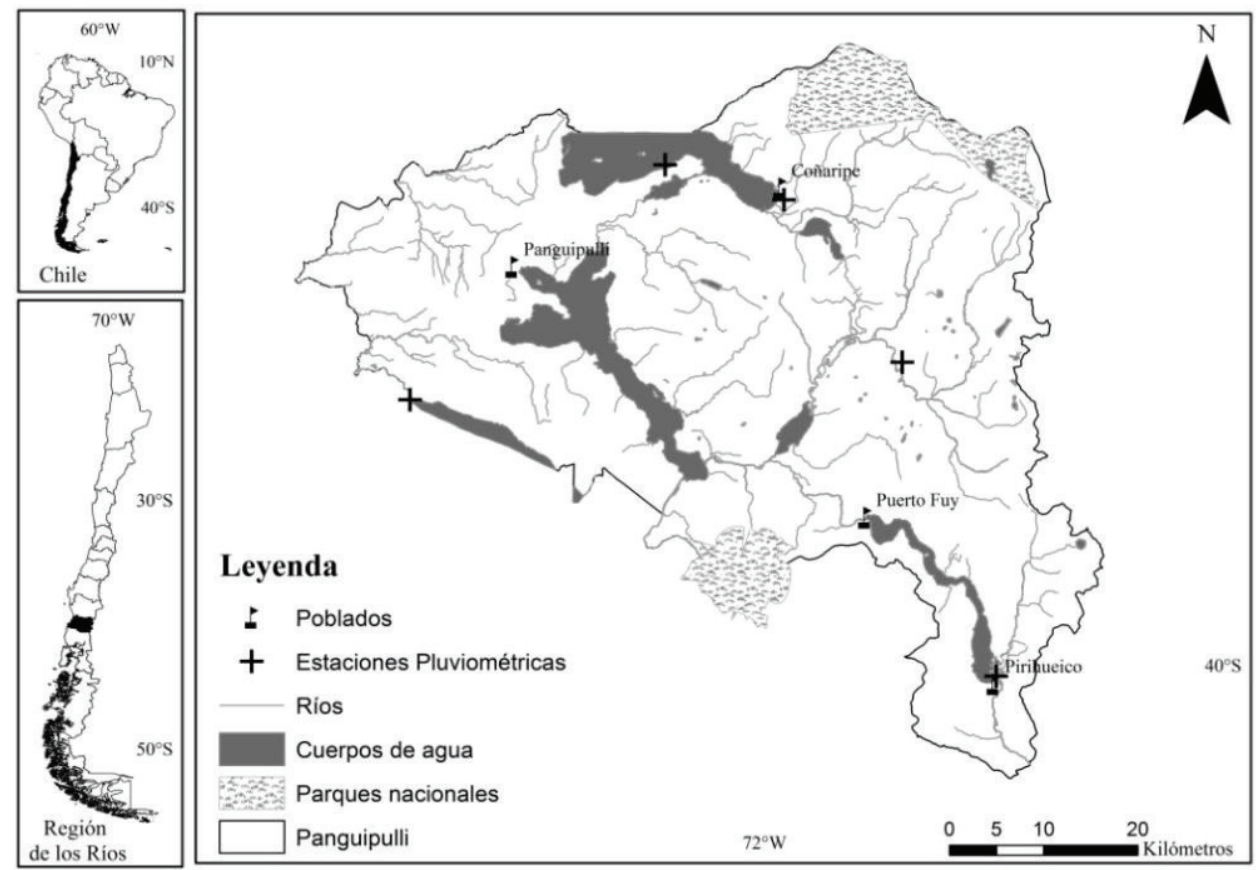

Figura 1. Área de estudio, localización de la región de Los Ríos. Principales poblados, estaciones pluviométricas, ríos, cuerpos de agua, parques nacionales y límites administrativos.

Study area in Los Ríos Region, southern Chile, showing urban areas, pluviometric stations, rivers, water bodies, national parks and administrative boundaries. 
Paso 1: Reclasificación de usos y coberturas del suelo. Para describir el uso del suelo, se utilizó la información espacial generada a través de la Actualización del Catastro de Uso Vegetacional para la Región de los Ríos, 2013, realizado por la Corporación Nacional Forestal (CONAF 2013). Esta información fue reclasificada a base de la siguiente categoría de coberturas del suelo: 1) Bosque Nativo, 2) Cultivo, 3) Matorral, 4) Matorral Arborescente, 5)
Plantación adulta, 6) Pradera, 7) Renoval y 8) Otros tipos (v.g., cuerpos de agua, ciudades). Finalmente, se generó una capa raster, donde el valor de cada pixel representa la cobertura del suelo en particular.

Paso 2: Determinación de grupos hidrológicos de suelo. Para determinar los grupos hidrológicos, se utilizó la información Series de Suelo generada por el Centro de Infor-
- Cobertura de usos del suelo (CONAF 2013).

- Series de suelo (CIREN 1999).

- Mapas históricos series suelo.

- Muestras de suelo en terreno.

- Valores CN (SCS).

- Consulta a tres expertos.

Pendiente ponderada (\%) por clases de CN (Ebrahimian et al. 2012).

Condición antecedente de humedad del suelo III en periodo de latencia (Boughton 1989).
- Registros pluviométricos (1998 2013) máximos mensuales en 24 horas.

- Normas y leyes referentes protección de recursos hídricos y bosque nativo. - Normas y leyes referentes a potencial expansión de plantaciones forestales.

- Límites cuencas hidrográficas.
Paso 1: Reclasificación de usos y coberturas de suelo.

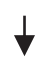

Paso 2: Determinación de grupos hidrológicos del suelo

Paso 3: Validación de valores de $\mathrm{CN}$ por consulta a expertos

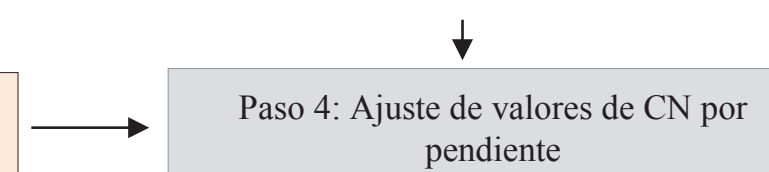

pendiente
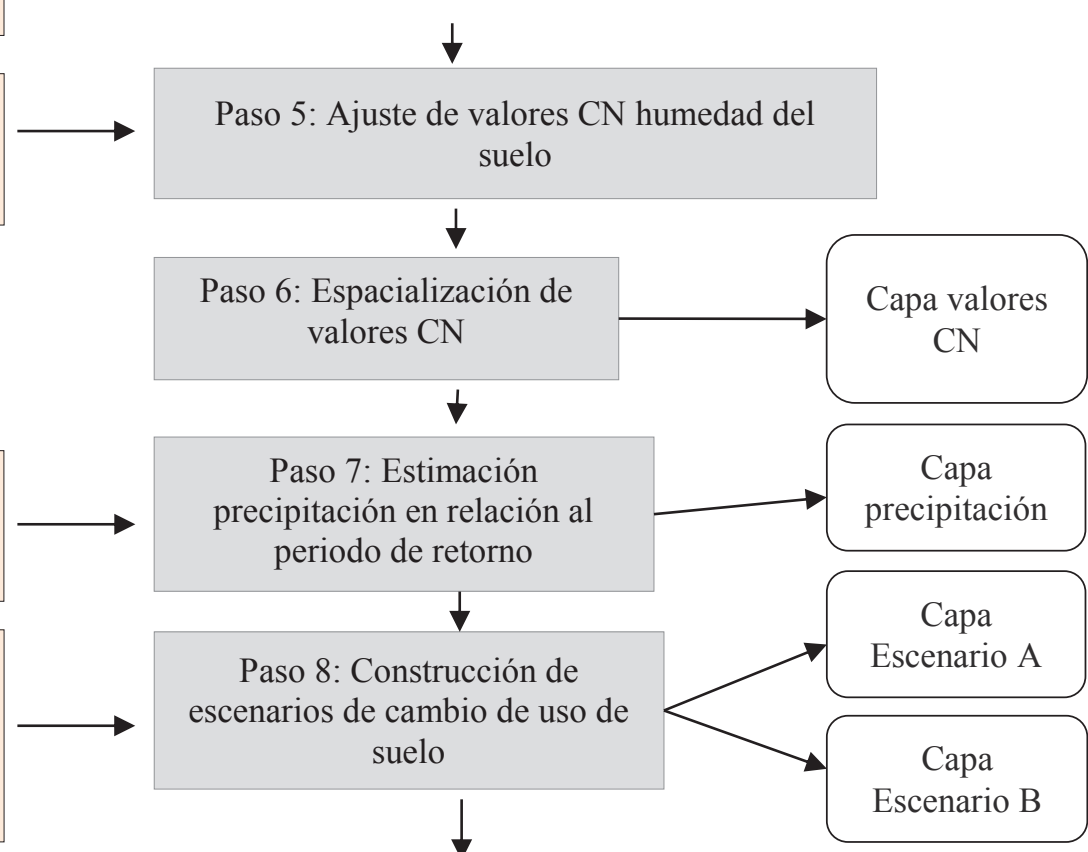

Paso 9: Mapeo del SE de regulación hídrica usando ECOSER
Insumo

Paso metodológico

Resultado

Flujo metodológico

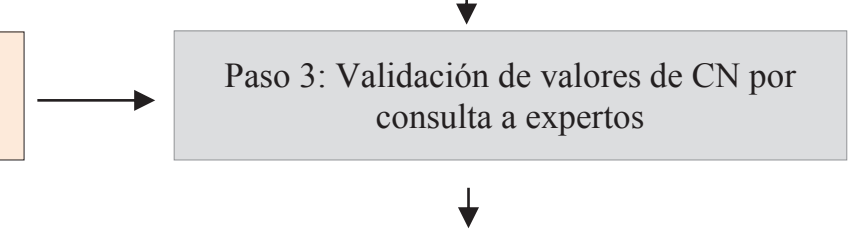


mación de Recursos Naturales para la Región de los Lagos (incluida la actual Región de los Ríos) (CIREN 1999). Esta información proporcionada por CIREN se encontraba incompleta, faltando la descripción de las clases de suelo en los sectores de la alta cordillera. Para completar la información se recurrió a mapas históricos de suelo, consulta de tres expertos de la Universidad Austral de Chile y Universidad de Concepción, y la toma de muestras de suelo en terreno en localidades cordilleranas de la comuna para completar pequeños sectores sin información. Se completó así una capa de información que contiene propiedades físicas de los suelos (i.e. textura, drenaje interno y profundidad) las cuales permitieron determinar a qué grupo hidrológico (i.e. A, B, C, D) correspondía cada serie de suelo. Los grupos hidrológicos comprenden desde el grupo A, que corresponden a suelos de texturas gruesas, con buen drenaje y profundos hasta el grupo $\mathrm{D}$, que corresponden a suelos de texturas finas, delgados, y con drenaje limitado. Además, se clasificaron dos grupos hidrológicos adicionales; i) Aw que representa los cuerpos de agua (lagos, lagunas y ríos) y ii) Dr que representa las zonas donde existen afloramientos rocosos o zonas desprovistas de vegetación. Finalmente se generó una capa raster, donde el valor de cada pixel representa el grupo hidrológico al cual pertenece.

Paso 3: Validación de valores $C N$ por medio de consulta a expertos. A base de los valores CN propuestos por el Departamento de Agricultura de los Estados Unidos (USDA 1989) para zonas del hemisferio norte, se realizaron consultas a expertos del área de la hidrología y edafología, de las Facultades de Ciencias Forestales y Recursos Naturales y Ciencias Agrarias de la Universidad Austral de Chile y Universidad de Concepción (tres expertos en total) lo que permitió validar y ajustar los valores de acuerdo a la zona de estudio. Cabe señalar que USDA (1989) no reporta valores de $\mathrm{CN}$ para plantaciones, razón por la cual los valores de bosque nativo sirvieron como punto de partida para generar dichos valores. Si bien la intensión original fue separar plantaciones jóvenes y adultas, se consideró que solo los valores de plantaciones adultas podían ser aproximados con mayor precisión. Los expertos pudieron estimar valores $\mathrm{CN}$ para plantaciones adultas a base de su conocimiento en estudios locales, ya que, en general, los estudios hidrológicos relacionados a plantaciones forestales en Chile utilizan plantaciones en su estado adulto para evaluar cambios eco-hidrológicos.

Paso 4: Ajuste de los valores de CN por pendiente. Los valores obtenidos del paso anterior, se ajustaron en función de la pendiente, con el fin de ponderarla como una variable que influye en la capacidad de regulación. Para esto, se intersectó una capa de pendientes (obtenida desde un Modelo Digital de Elevación) con la capa CN sin ajustar. Cada categoría/valor de $\mathrm{CN}$, se representó a través de polígonos en un archivo formato shapefile. En cada uno de estos polígonos, se identificaron distintos grados de pendiente, por lo que se calculó la pendiente ponderada en cada uno de ellos a través de la ecuación [1] (Ebrahimian et al. 2012). Finalmente, con la pendiente ponderada, se ajustó los valores $\mathrm{CN}$ utilizando la ecuación [2] y [3] (Ebrahimian et al. 2012).

$$
P P=\frac{\sum_{i-1}^{n} a_{i}^{*} s_{i}}{A}
$$

Donde,

$P P=$ pendiente ponderada (\%), $a_{i}=$ área de la pendiente (ha), $s_{i}=$ pendiente (\%), $A$ = área del polígono $\mathrm{CN}$ (ha).

$$
C N_{2 \alpha}=C N * K
$$

Donde, $C N_{2 \alpha}=$ valor $\mathrm{CN}$ corregido por pendiente, $C N=$ valor $\mathrm{CN}, K=$ constante para cada $C N_{2}$.

A su vez, la constante $K$ se calculó con la ecuación [3]

$$
K=\frac{322,79+15,63 * P P}{P P+323,52}
$$

Donde, $K=$ constante para cada $C N_{2}, P P=$ pendiente ponderada $(\%)$.

Paso 5: Ajuste de valores $C N$ por condición previa de humedad del suelo. Los valores validados y ajustados por pendiente, fueron ajustados por la condición de humedad del suelo, considerando la precipitación acumulada $(\mathrm{mm})$ en los cinco días previos al evento. Debido a que la precipitación a base de periodos de retorno (ver paso 7) no corresponde a un evento en particular, se identificaron eventos de rangos similares a estos eventos estadísticamente posibles, determinando la acumulación de cinco días previos para cada uno estos en particular. Finalmente, se promediaron los valores acumulados de cada evento dentro del rango, obteniendo un valor medio de acumulación y la época del año en que ocurrían (invierno o verano). El valor indica en qué condición de humedad se encuentra el suelo, con respecto a los rangos propuestos por Boughton (1989). En este caso se determinó una CHS III (Boughton 1989) y el período analizado se consideró en estado de latencia (época invernal). Así, se ajustaron los valores $\mathrm{CN}$ ya ajustados por pendiente, utilizando la ecuación [4] (Ahmad et al. 2015) para una CHS III.

$$
C N_{I I I}=\frac{\mathrm{CN}_{2 \alpha}}{\left(0,427+0,00573 * C N_{2 \alpha}\right)}
$$

Donde, $C N_{I I I}=$ valor $\mathrm{CN}$ bajo condiciones de humedad, $C N_{2 \alpha}=$ valor $\mathrm{CN}$ ajustado por pendiente.

Paso 6: Espacialización de los valores CN. Para espacializar los valores $\mathrm{CN}$, se asignaron valores numéricos a las capas Uso Actual y Grupo Hidrológico. Con esto, se creó 
una capa preliminar a través de la herramienta raster calculator, donde cada pixel representa el valor del tipo de uso de suelo y el grupo hidrológico en el cual se sitúa esa cobertura de suelo en particular. Finalmente, a los pixeles de la capa resultante, se les asignaron los valores $\mathrm{CN}$ ajustados.

Paso 7: Estimación de la precipitación en relación al periodo de retorno. Se llevaron a cabo análisis estadísticos para determinar rangos de tormentas máximas en 24 horas, con su respectiva probabilidad de ocurrencia y periodo de retorno en dos años. Esto se realizó a través de estadística sobre eventos extremos ajustado por la distribución de Gumbel, por medio de datos de precipitación acumulada en 24 horas, registrados desde 1998 hasta 2013 por la Dirección General de Aguas (DGA) en 10 estaciones de observación de las comunas de Lanco, Lago Ranco, Pucón y Panguipulli. Para determinar cómo se distribuye la precipitación en el área de estudio, se generó una capa raster a través del método de interpolación geo-estadística kriging por medio de puntos geoespaciales (estaciones) que informan rangos de precipitación sobre períodos de retorno en dos años. Así, el valor de cada pixel corresponde al rango de precipitación que caería $(\mathrm{mm})$ con una probabilidad de ocurrencia de $50 \%$ en dos años.

Paso 8: Construcción de escenarios de cambio de uso del suelo. Se construyeron dos escenarios de cambio de uso de suelo (A y B) a través de la combinación de reglas heurísticas y aplicación de la legislación chilena en cuanto a la protección de bosque nativo, recursos hídricos y las exigencias del Decreto Ley $\mathrm{N}^{\circ} 701$ de subsidio a plantaciones, contenido en la Ley 20283 sobre recuperación del bosque nativo y fomento forestal. Esta información fue transformada a insumos geo-espaciales con el fin construir los escenarios.

- Escenario A, conservación de bosque nativo. Para la construcción de este escenario se establecieron las siguientes reglas heurísticas con respecto a las áreas probables de conservación y recuperación del bosque nativo: a) la permanencia de iniciativas de conservación presentes en la comuna (i.e. Reserva privada San Pablo de Tregua, Parque Nacional Villarrica, Reserva Nacional Mocho-Choshuenco, Reserva privada Huilo-Huilo), b) restauración de zonas de amortiguación de franjas ribereñas contemplando $200 \mathrm{~m}$ de protección vegetal por cada ribera de manantiales (agua que brota de las cimas de laderas y cerros) y 20 metros para los cuerpos de agua en general, regulación contenida en la Ley 20283 de bosque nativo y c) protección del bosque nativo adulto a través de la Zona de Interés Turístico para Panguipulli (ZOIT) (Romero et al. 2014). Esta información fue espacializada y asignada como una cobertura de bosque nativo adulto. Se asumió que para obtener bosque nativo adulto desde coberturas como praderas, matorrales y matorrales arborescentes, se emplearía una reforestación con especies pioneras (v.g., Nothofagus obliqua Mirb. Oerst, Nothofagus dombeyi Mirb. Oerst, Embothrium coccineum J.R. Forst et G. Forst). Para el caso de coberturas de plantación forestal y cultivo, se debe realizar raleos selectivos para disminuir la cobertura de copas y permitir mayor ingreso de luz, con el fin de que especies pioneras comiencen a repoblar el área. Además, el raleo selectivo impide la colonización masiva de Chusquea quila Kunth y se deberá hacer hasta extraer todos los individuos exóticos, para posteriormente pasar una restauración activa aplicando criterios señalados anteriormente. En el caso del bosque renoval, se debe realizar raleos ecológicos, extrayendo individuos en un estado de desarrollo Latizal alto y bajo, con el fin de que el bosque adquiera una estructura de bosque adulto y estado de desarrollo fustal- maduro.

- Escenario B, aumento de plantaciones forestales exóticas: Para generar este escenario, se utilizó como base: a) las trayectorias pasadas de cambio de uso de suelo en la comuna y b) la distribución potencial de plantaciones forestales que la legislación de Chile permite. Las trayectorias de cambio de uso de suelo nos indican las tendencias entre los usos del suelo y cuáles de estos han sido transformados a plantaciones forestales históricamente. Usos como cultivo, pradera, matorral y matorral arborescente, han sido transformados al uso plantación adulta. A base de esto, a los usos del suelo mencionados se les asignó la cobertura "Plantación adulta", la cual representa plantaciones forestales de especies exóticas (v.g., Eucalyptus nitens, Pinus radiata), adultas (10 - 20 años). Los usos como bosque nativo y bosque renoval son usos en donde las plantaciones forestales exóticas no puede ser establecidas, por ende no cambian.

Paso 9: Mapeo del servicio ecosistémico de regulación hídrica usando ECOSER. Para generar el proxy del servicio ecosistémico de regulación hídrica, se utilizó la extensión ECOSER en Arcgis 10.1, que permite utilizar las siguientes ecuaciones:

$$
I E=\frac{25400}{C N_{2 \alpha}}-254
$$

Donde, $I E$ = infiltración y evapotranspiración (mm), $C N_{2 \alpha}$ $=$ valor $\mathrm{CN}$ ajustado por pendiente.

$$
R E P=P-\frac{(P-0,2 * I E)^{2}}{(P+0,8 * I E)} * n^{\mathrm{o}} \text { tormentas }
$$

Donde, $R E P$ = retención de exceso de precipitación $(\mathrm{mm})$, $I E=$ infiltración y evapotranspiración $(\mathrm{mm})$,

$P=$ precipitación $(\mathrm{mm})$. 
Finalmente, el mapeo del servicio ecosistémico mediante ECOSER se llevó a cabo utilizando como insumos, la capa de valores $\mathrm{CN}$ espacializados para el año base, los escenarios y la precipitación en función de los periodos de retorno utilizada para los tres análisis (año base y escenarios). Con esta información fue posible aplicar la ecuación [5] y ecuación [6], obteniendo como resultado tres capas espacializadas (i.e. año base, escenario A y escenario B), donde el valor de cada pixel representa su capacidad de regulación medida en milímetros (mm). Para obtener los valores del servicio ecosistémico para cada cuenca, se extrajo la información a través de la herramienta extract by mask, la cual permitió extraer información desde un archivo raster a través de un shapefile (cuencas), para cada una de las 16 cuencas estudiadas (figura 3). Los resultados obtenidos en milímetros fueron transformados a metros cúbicos considerando que $1 \mathrm{~mm}$ equivale a $0,001 \mathrm{~m}$ de agua. Así, multiplicado por la superficie $\left(\mathrm{m}^{2}\right)$, se obtiene el valor en metros cúbicos. Para establecer cambios ante los escenarios, se utilizó el valor medio $(\overline{\mathrm{X}})$ que involucra el promedio entre las 16 cuencas analizadas y/o parte de ellas.

\section{RESULTADOS}

Valores $C N$. Los valores $\mathrm{CN}$ obtenidos a través de la consulta a expertos e información previa (valores $\mathrm{CN}$ generados por SCS) mostraron que en general, los valores más bajos (mayor regulación) correspondieron a coberturas del tipo Bosque nativo y Renoval, sobre grupos hidrológicos A y B. Al contrario, los valores más altos (menor regulación), se asociaron a coberturas del tipo Plantación adulta, Pradera y Cultivo, sobre los grupos C y D. El valor para el grupo $A w$ es 1, independiente de la cobertura, debido a que este grupo representa los cuerpos de agua, los cuales retienen el total del agua caída ante un evento de tormenta. Al contrario, el grupo Dr, se asocia a valores cercanos a 100 , ya que este grupo representa las zonas de afloramientos rocosos y zonas desprovistas de vegetación, donde la capacidad de regulación es muy baja o nula (cuadro 1).

Precipitación: Periodos de retorno sobre máximos diarios mensuales. La interpolación de Kriging mostró, espacialmente, que la distribución pluviométrica no es homogénea en el paisaje. La pluviometría fue menor en localidades situadas

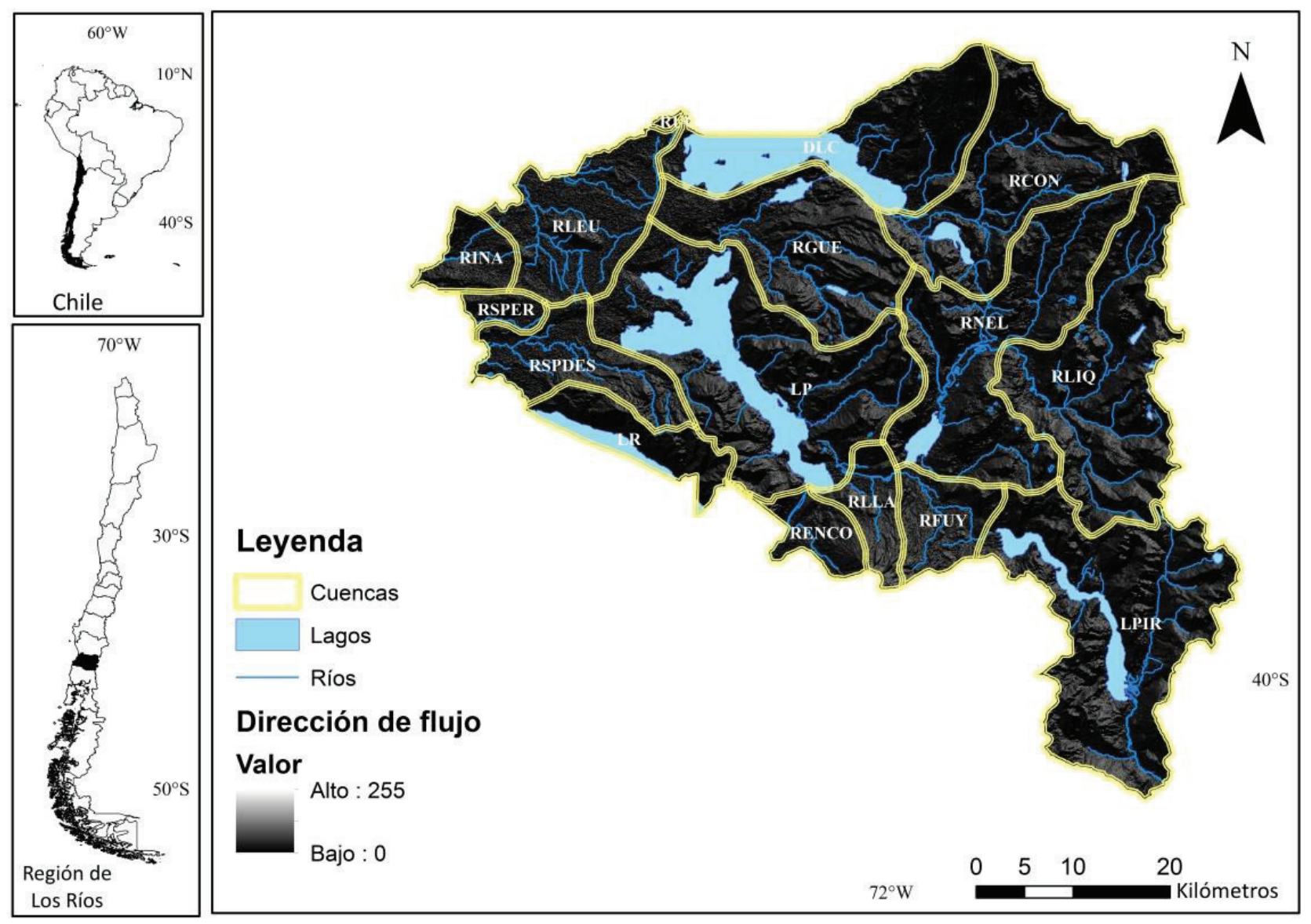

Figura 3. Cuencas hidrográficas de la comuna de Panguipulli, ríos, cuerpos de agua y dirección de flujo.

Watersheds of the municipality of Panguipulli, rivers, water bodies and flow direction. 
en la depresión intermedia $(52,7 \mathrm{~mm})$, alrededor de los 290 m s.n.m. Aumenta sostenidamente en dirección Oeste-Este, a medida que la geografía cambia hacia sectores dominados por cerros y cumbres $(1.000-1.700 \mathrm{~m}$ s.n.m. $)$ y valles meridionales de la pre-codillera y cordillera andina, donde ocurren eventos de tormenta que podrían alcanzar el orden de los 129,09 mm caídos en 24 horas con una probabilidad de $50 \%$. El agua que caería en la comuna alcanza los $315.978 .230 \mathrm{~m}^{3}$.

Escenarios de uso de suelo. En general, los usos Bosque nativo y Plantación adulta predominarían en superficie, tanto para el año base (2013) como en los escenarios (figura 4). En el escenario A, el uso Bosque nativo au-

Cuadro1. Valores CN validados y corregidos por pendiente y condición de humedad del suelo.

Curve number values validated and corrected by slope and soil moisture condition.

\begin{tabular}{lcccccc}
\hline \multirow{2}{*}{ Uso de suelo } & \multicolumn{7}{c}{ Grupo hidrológico } \\
\cline { 2 - 7 } & Aw & $\mathrm{A}$ & $\mathrm{B}$ & $\mathrm{C}$ & $\mathrm{D}$ & $\mathrm{Dr}$ \\
\hline Bosque nativo & 1 & 40 & 56 & 63 & 76 & 99 \\
Cultivo & 1 & 58 & 61 & 73 & 75 & 99 \\
Matorral & 1 & 50 & 59 & 70 & 75 & 99 \\
Matorral arborescente & 1 & 46 & 54 & 58 & 65 & 99 \\
Plantación adulta & 1 & 73 & 89 & 91 & 96 & 99 \\
Pradera & 1 & 81 & 89 & 93 & 95 & 99 \\
Renoval & 1 & 56 & 69 & 75 & 78 & 99 \\
Otros tipos & 1 & 66 & 68 & 70 & 71 & 99 \\
\hline
\end{tabular}

mentaría de 51 a $82 \%$ respecto del año base, debido a que los usos Cultivo, Matorrales y Pradera cambiarían a este uso, mientras que en el escenario $\mathrm{B}$ el uso Bosque nativo se mantendría al año base. Por otro lado, en el escenario A la superficie del uso Plantación adulta sería similar al año base mientras que en el escenario B las plantaciones aumentarían de 15 a $20 \%$ respecto del año base (cuadro 2 ).

Efecto del cambio de uso de suelo en el servicio ecosistémico de regulación hídrica. Los resultados mostraron que los valores máximos ocurrirían en el escenario A, mientras que los valores medios, se obtendrían para el año base (2013) y los valores más bajos en el escenario B.

En términos generales, en el escenario A la capacidad de regulación hídrica aumentó en $12.303 .222 \mathrm{~m}^{3} \mathrm{y}$ mientras que en el escenario B disminuyó en $5.232 .886 \mathrm{~m}^{3}$. La diferencia de magnitud entre la ganancia y pérdida del servicio ecosistémico entre escenarios, se debió a que en el escenario A, el bosque nativo adulto aumentó en mayor proporción que las plantaciones forestales exóticas en el escenario B (117.000 y 56.000 ha, respectivamente).

Efectos de los escenarios a escala de cuenca hidrográfica. Considerando como referencia el año base (2013), en el escenario A las 16 cuencas de la comuna aumentarían su capacidad de regulación hídrica $\left(\overline{\mathrm{x}}=8,51 \mathrm{~m}^{3} \mathrm{ha}^{-1}\right)$, mientras que ante el escenario $\mathrm{B}$, todas las cuencas mermarían su capacidad de regulación hídrica $\left(\overline{\mathrm{X}}=3,1 \mathrm{~m}^{3} \mathrm{ha}^{-1}\right)$.

En el escenario A, nueve de las 16 cuencas (i.e. LR, RCRU, RFUY, RGUE, RLLA, RLEU, RINA, RSPDES, RSPER) aumentarían considerablemente su capacidad de regulación hídrica por unidad de superficie (sobre la media) $\left(\overline{\mathrm{X}}=11,18 \mathrm{~m}^{3} \mathrm{ha}^{-1}, \mathrm{~s}^{2}= \pm 1,82 \mathrm{~m}^{3} \mathrm{ha}^{-1}\right)$, con valores máximos y mínimos de $14,59 \mathrm{~m}^{3} \mathrm{ha}^{-1}$ y $9,26 \mathrm{~m}^{3} \mathrm{ha}^{-1}$, res-
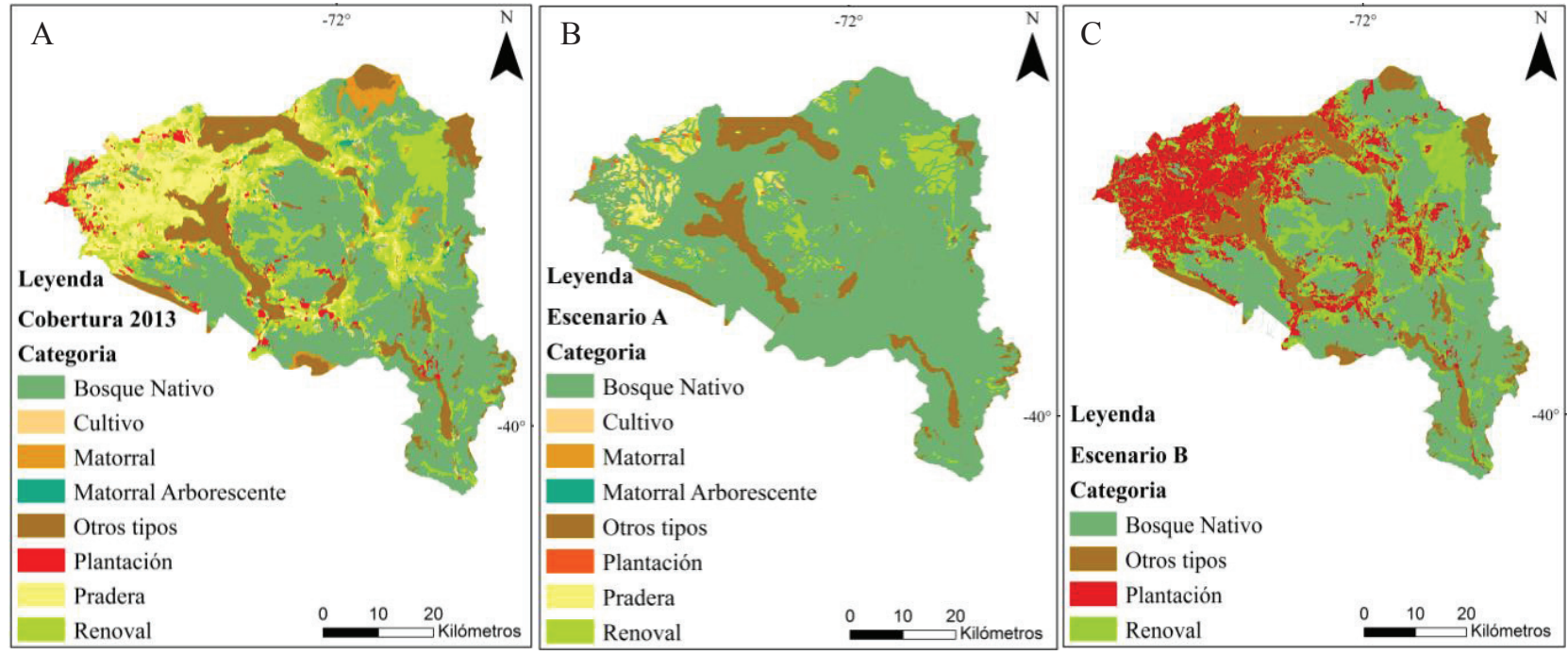

Figura 4. (A) Distribución espacial de los usos del suelo para el año base 2013. (B) Escenario A. (C) Escenario B. Spatial distribution of land uses for: (A) Base year 2013. (B) Scenario A. (C) Scenario B. 
pectivamente. Gran parte de la superficie donde se expandiría el bosque nativo corresponde a praderas y plantaciones forestales ( $\bar{X}=41,5 \%$ y $\bar{X}=24,8 \%$, respectivamente) $\mathrm{y}$ en menor proporción a matorrales y matorrales arborescentes $(\overline{\mathrm{X}}=1,22 \%$ y $\overline{\mathrm{X}}=0,39 \%)$.

Por otro lado, las siete cuencas restantes (i.e. DLC, LP, LPIR, RCON, RENCO, RLIQ, RNEL) aumentarían levemente su capacidad de regulación (bajo la media) $\left(\bar{X}=4,88 \mathrm{~m}^{3}\right.$ $\mathrm{ha}^{-1}, \mathrm{~s}^{2}= \pm 2,67 \mathrm{~m}^{3} \mathrm{ha}^{-1}$ ) con un valor máximo y mínimo de
7,67 $\mathrm{m}^{3} \mathrm{ha}^{-1}$ y $1,33 \mathrm{~m}^{3} \mathrm{ha}^{-1}$, respectivamente. En estas cuencas, la superficie que sería cedida al bosque nativo provendría en gran parte de bosques renovales $(\overline{\mathrm{X}}=19,2 \%)$, praderas $(\bar{X}=14,71 \%)$, matorrales $(\bar{X}=4,43 \%)$ y en menor proporción de plantaciones forestales $(\overline{\mathrm{X}}=1,48 \%$ ) (figura 5).

En el escenario B, cinco de las 16 cuencas (i.e. LPIR, RCON, RGUE, RLIQ, RNEL), disminuirían considerablemente su capacidad de regulación hídrica (sobre la media) $\left(\overline{\mathrm{X}}=-4,45 \mathrm{~m}^{3} \mathrm{ha}^{-1}, \mathrm{~s}^{2}= \pm 0,93 \mathrm{~m}^{3} \mathrm{ha}^{-1}\right)$ con un valor máximo

Cuadro 2. Superficies de las distintas coberturas y usos de suelo para el año base 2013 y los escenarios A y B. Areas of the different land cover and uses for base year 2013 and scenarios A and B.

\begin{tabular}{lrrrrrr}
\hline \multirow{2}{*}{ Uso de suelo } & \multicolumn{2}{c}{2013} & \multicolumn{2}{c}{ Escenario A } & \multicolumn{2}{c}{ Escenario B } \\
\cline { 2 - 7 } & Área (ha) & $\%$ & Área (ha) & $\%$ & Área (ha) & $\%$ \\
\hline Bosque nativo & 167.355 & 51 & 270.605 & 82 & 167.355 & 51 \\
Cultivo & 2.939 & 1 & 476 & - & - & - \\
Matorral & 5.017 & 2 & 1.042 & - & - & - \\
Matorral arborescente & 3.276 & 1 & 311 & - & - & - \\
Plantación adulta & 49.447 & 15 & 31.591 & 10 & 64.766 & 20 \\
Pradera & 2.904 & 1 & 1.552 & - & - & - \\
Renoval & 53.904 & 16 & 8.846 & 3 & 56.364 & 17 \\
Otros tipos & 45.067 & 14 & 14.195 & 4 & 43.274 & 13 \\
\hline
\end{tabular}

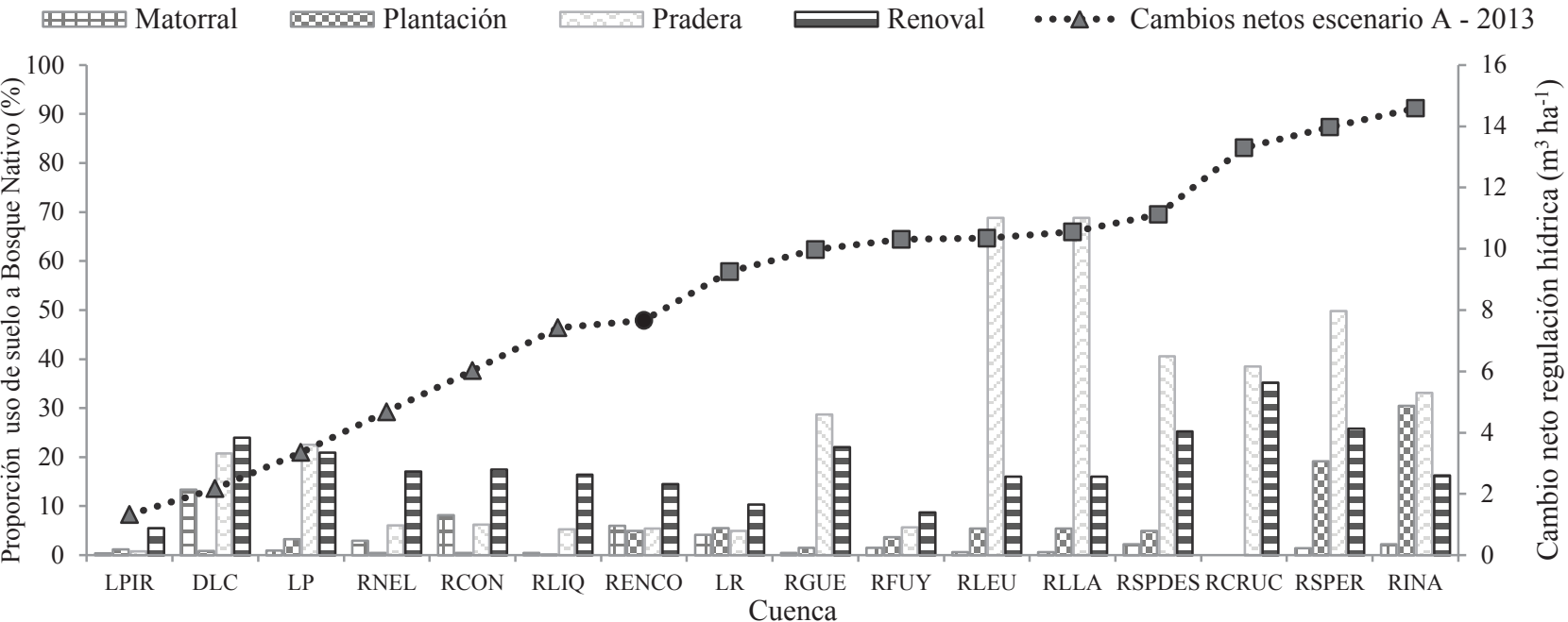

Figura 5. Cambios netos en el servicio ecosistémico de regulación hídrica para año base y escenario A en las 16 cuencas ordenadas de menor a mayor aumento y porcentaje de usos del suelo que pasarían al uso bosque nativo. Marcador cuadrado indica valor sobre la media, marcador triangular indica valor bajo la media y marcador circular marca la media.

Net changes in water regulation for the base year and scenario A in the 16 basins ranked from the lowest to the highest increase, and percentage of land uses that would pass to native forest use. Square markers indicate values above the mean, triangular markers indicate values below the mean and circular markers indicate mean values. 
Cuadro 3. Distribución en porcentaje de grupos hidrológicos en cada cuenca analizada con respectiva capacidad de regulación hídrica medida en metros cúbicos por hectárea.

Distribution in percentage of hydrological groups in each analyzed basin with respective water regulation capacity measured in cubic meters per hectare.

\begin{tabular}{|c|c|c|c|c|c|c|c|}
\hline \multirow{2}{*}{ Cuenca } & \multicolumn{6}{|c|}{ Grupo hidrológico (\%) } & \multirow{2}{*}{$\begin{array}{c}\text { Regulación } \\
\text { hídrica }\left(\mathrm{m}^{3} \mathrm{ha}^{-1}\right)\end{array}$} \\
\hline & Aw & $\mathrm{A}$ & $\mathrm{B}$ & $\mathrm{C}$ & $\mathrm{D}$ & $\mathrm{Dr}$ & \\
\hline LPIR & 3 & 46 & 41 & 0 & 0 & 10 & 608 \\
\hline RFUY & 7 & 41 & 32 & 0 & 0 & 20 & 604 \\
\hline RLIQ & 8 & 30 & 36 & 15 & 0 & 11 & 602 \\
\hline RNEL & 16 & 2 & 33 & 33 & 0 & 15 & 589 \\
\hline $\mathrm{RCON}$ & 12 & 0 & 28 & 30 & 9 & 20 & 582 \\
\hline RLLA & 20 & 35 & 0 & 37 & 1 & 7 & 572 \\
\hline RENCO & 20 & 35 & 15 & 16 & 4 & 10 & 568 \\
\hline $\mathrm{LC}$ & 9 & 14 & 0 & 32 & 20 & 15 & 531 \\
\hline LP & 15 & 12 & 19 & 30 & 18 & 6 & 528 \\
\hline RGUE & 18 & 14 & 20 & 21 & 21 & 7 & 519 \\
\hline LR & 5 & 15 & 21 & 22 & 20 & 17 & 505 \\
\hline RSPDES & 0 & 5 & 29 & 41 & 24 & 0 & 486 \\
\hline RCRUC & 0 & 30 & 0 & 40 & 30 & 0 & 486 \\
\hline RLEU & 0 & 10 & 33 & 28 & 30 & 0 & 464 \\
\hline RSPER & 10 & 11 & 15 & 27 & 32 & 5 & 448 \\
\hline RINA & 0 & 26 & 48 & 0 & 26 & 0 & 430 \\
\hline
\end{tabular}

de $-5,55 \mathrm{~m}^{3} \mathrm{ha}^{-1}$ y mínimo de $-3,31 \mathrm{~m}^{3} \mathrm{ha}^{-1}$. Además en estas cuencas, la superficie que cubrirían las plantaciones forestales provendría de forma considerable desde las praderas $(\bar{X}=69,07 \%)$, matorrales $(\bar{X}=14,1 \%)$ y matorrales arborescentes $(\overline{\mathrm{X}}=5,34 \%)$. Por otro lado, estas cuencas contenían baja cantidad de plantaciones forestales al año base $(\overline{\mathrm{X}}=5,02 \%)$.

Las 11 cuencas restantes (i.e. DLC, LP, LR, RCRU, RENCO, RFUY, RINA, RLEU, RLLA, RSPDES, RSPER), reducirían levemente su capacidad de retener agua (bajo la media) $\left(\overline{\mathrm{x}}=2,48 \mathrm{~m}^{3} \mathrm{ha}^{-1}, \mathrm{~s}^{2}= \pm 0,55 \mathrm{~m}^{3} \mathrm{ha}^{-1}\right)$ con un valor máximo de $3,36 \mathrm{~m}^{3} \mathrm{ha}^{-1}$ y mínimo de $1,69 \mathrm{~m}^{3} \mathrm{ha}^{-1}$. Por otro lado, gran parte de la superficie que cubriría las plantaciones forestales provendría principalmente desde praderas $(\overline{\mathrm{X}}=66,58 \%)$ y en menor proporción desde matorral y matorral arborescente $(\bar{X}=4,87 \%$ y $\bar{X}=5,93 \%)$. Además, gran parte de la superficie de estas cuencas estaba previamente (año base) cubierta por plantaciones forestales en el año base $(\bar{X}=22,38 \%$ ) (figura 6$)$.

\section{DISCUSIÓN Y CONCLUSIONES}

Para entender cómo el cambio de uso de suelo afecta e interactúa con el sistema ambiental, se necesita información sobre qué cambios ocurren, dónde y cuándo ocurren, las tasas de cambio y los efectos que causan en los servicios ecosistémicos.

A diferencia de otros estudios realizados en Chile, este estudio es innovador en los siguientes aspectos; i) no se enfoca en la oferta de agua sino en la regulación hídrica como servicio ecosistémico igualmente relevante; ii)

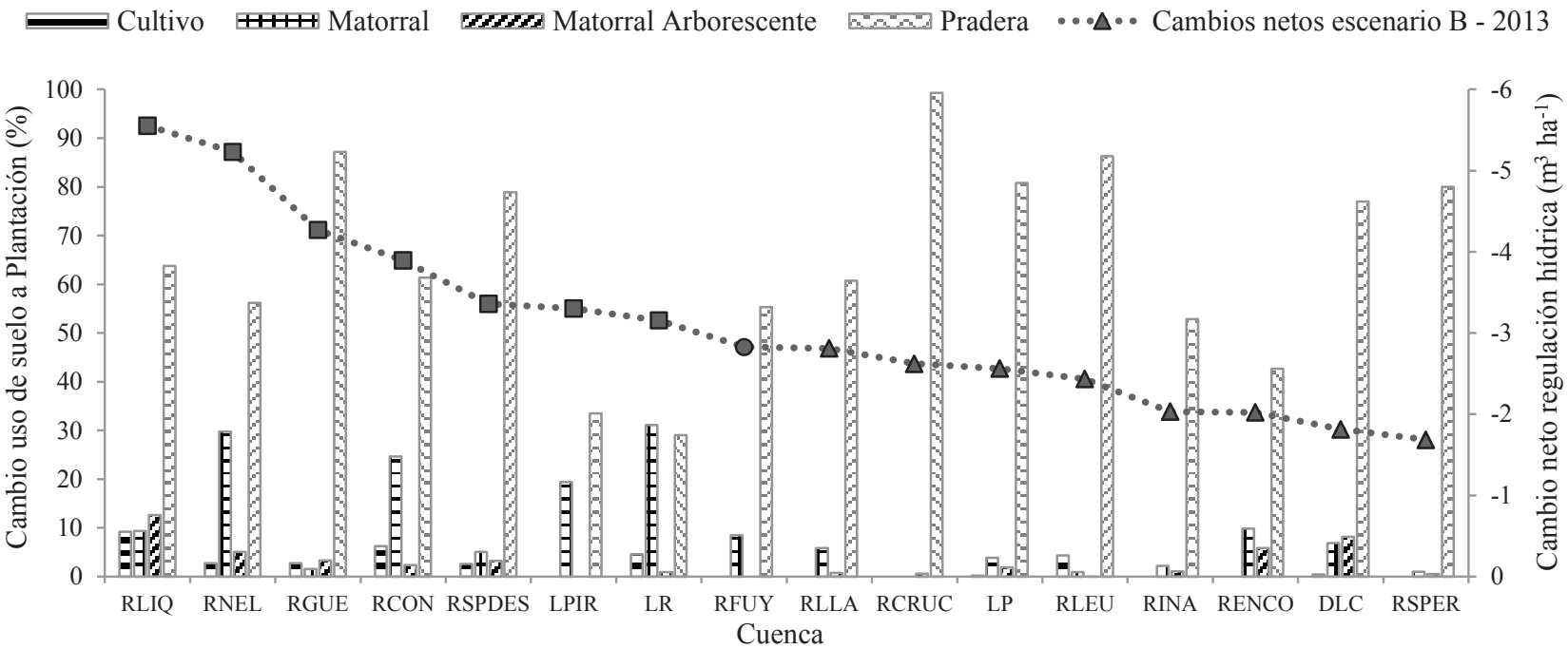

Figura 6. Cambios netos de servicio ecosistémico de regulación hídrica para año base y escenarios B en las 16 cuencas ordenadas de mayor a menor reducción y porcentaje de usos del suelo que pasarían al uso Plantación adulta. Marcador cuadrado indica valor sobre la media, marcador triangular indica valor bajo la media y marcador circular marca la media.

Net changes in water regulation for the base year and scenario B in the 16 basins ranked from the lowest to the highest increase, and percentage of land uses that would pass to exotic plantations use. Square markers indicate values above the mean, triangular markers indicate values below the mean and circular markers indicate mean values. 
se basa en una metodología nueva y ampliamente replicable (ECOSER); iii) aporta a precisar la diferencia entre provisión y regulación hídrica, donde esta última se define como la cantidad de agua que es captada e infiltrada a napas subterráneas dependiendo, en este caso, de la cobertura/uso de suelo y las propiedades físicas de suelo; iv) ayuda a comprender el rol eco-hidrológico del bosque nativo y las plantaciones de monocultivo exótico; y v) a diferencia de otros estudios realizados en Chile (Lara et al. 2009) que abordan de forma empírica y en pequeñas cuencas los servicios ecosistémicos, este estudio mapea el servicio ecosistémico a escala comunal, lo que posibilita evaluar los efectos del cambio de uso de suelo a dichas escalas (Saad et al. 2013) y ser un insumo acorde al modo en que la planificación territorial se aplica.

Los resultados del estudio otorgan indicios de los posibles efectos que puede generar el cambio de uso de suelo sobre la capacidad de regulación hídrica y por ende entregan información crítica para el manejo de recursos hídricos y planificación territorial en el área de estudio seleccionada. Los cambios cuantitativos en el servicio ecosistémico se atribuyen esencialmente al aumento del bosque nativo y de plantaciones forestales, como una manera de evaluar el efecto del cambio de uso de suelo en los servicio ecosistémico, como lo ha podido establecer Jaramillo (2014) con un estudio similar. Por otro lado, aunque aún existen restricciones metodológicas para definir tendencias y efectos del CUS en los servicios ecosistémicos (Kuczera 1987), investigaciones realizadas en diferentes países han podido establecer el efecto de plantaciones forestales y su efecto en la disminución de este servicios ecosistémico (Calder et al. 1997, Dean et al. 2015) en paralelo a otros trabajos que revelan la capacidad del bosque nativo como soporte para proveer este servicio ecosistémico (Licata et al. 2008; Jaramillo 2014) además del servicios ecosistémico de provisión de agua (Little et al. 2009, Little y Lara 2010).

Así, ante el escenario A, la superficie de bosque nativo aumenta en 117.000 ha aproximadamente, con cambios importantes en las zonas circunscritas a la depresión intermedia de la comuna. El servicio ecosistémico incrementó en $1.997 .423 \mathrm{~m}^{3}$ la cantidad de agua regulada. Desde el punto de vista eco-hidrológico, las plantaciones, cultivos y praderas son coberturas con muy baja capacidad de regulación hídrica en comparación al bosque nativo adulto, lo que explica que en las cuencas donde el bosque nativo se superpondría de forma considerable sobre usos como praderas $(\bar{X}=37,7 \%)$, cultivos $(\bar{X}=1,04 \%)$ y plantación adulta $(\bar{X}=8,48 \%)$ se condiga con los valores más altos de aumento neto en el servicio ecosistémico $\left(\overline{\mathrm{X}}=11,5 \mathrm{~m}^{3} \mathrm{ha}^{-1}\right)$. Las cuencas que muestran valores medios de cambio neto son aquellas donde la proporción de bosque nativo se mantiene $(\bar{X}=64,8 \%)$, lo que explica que el aumento de estas no sea considerable. Además, gran parte del aumento de bosque nativo reemplazaría bosques de renoval $(\bar{X}=16,9 \%)$, con similares capacidades de regulación. Por otro lado, las cuencas que muestran cambios netos leves
$\left(\bar{X}=4,88 \mathrm{~m}^{3} \mathrm{ha}^{-1}\right)$, mantienen gran parte de su superficie de bosque nativo ante el escenario A $(\bar{X}=59,2 \%)$. De la superficie que cambia, gran proporción provendría desde el bosque renoval $(\overline{\mathrm{X}}=16,8 \%)$, con similares capacidades de regulación y en menor proporción desde las praderas $(\bar{X}=14,7 \%)$ con menor capacidad de regulación.

En el escenario B, la superficie de plantación adulta aumenta en 56.000 ha, aproximadamente, particularmente sobre praderas, cultivos y matorrales en zonas de la depresión intermedia de la comuna, disminuyendo en $1.163 .624 \mathrm{~m}^{3}$ la cantidad de agua regulada. Desde el punto de vista eco-hidrológico, las plantaciones forestales producen mayores tasas de evapotranspiración y consumo de agua para su crecimiento en comparación a usos como matorral, matorral arborescente y pradera (Huber et al. 2008), exceptuando a los cultivos que muestran mayores tasas de evapotranspiración (Chen et al. 2010). La expansión de plantaciones forestales se situaría de forma considerable sobre el uso pradera $(\bar{X}=65,3 \%)$. Sin embargo, lo que explica que ciertas cuencas muestren pérdidas considerables de capacidad de regulación hídrica se condice con que la expansión forestal restante (excluyendo la que sustituiría las praderas) ocurriría sobre usos como matorral ( $\overline{\mathrm{X}}=5,86 \%)$ y matorral arborescente $(\bar{X}=16,3 \%)$, los cuales permiten mayor capacidad de regulación que las especies de monocultivo forestal. Además, estas cuencas muestran que muy poca proporción de su superficie está cubierta por plantaciones forestales para el año base $(\bar{x}=5,02 \%)$. Por otro lado, las cuencas que muestran pérdidas leves del servicio ecosistémico evidencian a su vez menor proporción de sustitución de plantaciones en los usos matorral ( $\overline{\mathrm{X}}=5,1 \%$ ) y matorral arborescente $(\overline{\mathrm{X}}=3,16 \%)$ ante el escenario. Además, estas cuencas muestran que gran parte de su superficie ya se encuentra colonizada por plantaciones forestales $(\bar{X}=25,91 \%)$, previo a la evaluación del escenario, lo que explica la poca variación.

Por otro lado, con respecto a los suelos como variable de importancia, se observa que las cuencas con los valores más altos de regulación hídrica $\left(>532,6 \mathrm{~m}^{3} \mathrm{ha}^{-1}\right)$ poseen gran parte de su superficie con suelos del tipo A (27,1\%) y B (26,5\%) con una capacidad de regulación en promedio de $589,3 \mathrm{~m}^{3}$ $\mathrm{ha}^{-1}$. Estos grupos hidrológicos representan suelos de texturas muy gruesas a gruesas (v.g., arenas gruesas y finas), muy profundos a profundos y con muy buen drenaje interno, lo que permite mayor capacidad de regulación hídrica. En contraste, se puede observar que para el caso de las cuencas con la menor capacidad de regulación $\left(<532,6 \mathrm{~m}^{3} \mathrm{ha}^{-1}\right)$, posee gran proporción de su superficie cubierta con suelos tipo C $(28,9 \%)$ y D $(27,8 \%)$ con una capacidad de regulación hídrica en promedio de 488,5 $\mathrm{m}^{3} \mathrm{ha}^{-1}$. Estos grupos hidrológicos representan suelos de texturas medias a finas (v.g., limo, arcilla), poco profundos a delgados y con drenaje restringido, lo que permite una menor capacidad de regulación hídrica. Estos resultados no consideran las relaciones con los escenarios ya que los grupos hidrológicos no 
muestran cambios ante estos, aunque si explica la distribución heterogénea de los cambios en el paisaje.

De acuerdo con los resultados, las áreas más expuestas al cambio de uso de suelo futuro serían aquellas ubicadas donde las plantaciones colonizan matorrales y matorrales arborescentes que también han experimentado escasez hídrica o donde eventualmente aumentaría el bosque nativo por sobre plantación adulta, praderas y cultivos. Estas áreas ya están experimentando una rápida transformación a plantaciones que coexisten con múltiples trayectorias terrestres de deforestación y degradación forestal. Por ende, resulta relevante aumentar o al menos mantener la masa boscosa nativa precisamente en estas áreas, con el fin de otorgar mayor capacidad para enfrentar la escasez hídrica a través del aumento en la capacidad de regulación hídrica.

La planificación puede ser muy difícil en estos paisajes dinámicos, especialmente, cuando los procesos de cambio del paisaje no están regulados, como es el caso de las zonas rurales en Chile, donde la mayoría de los instrumentos de política son indicativos y no normativos. Por lo tanto, la planeación eficaz de la expansión de las plantaciones, para evitar impactos negativos, requerirá muy probablemente una combinación de incentivos de mercado por un lado (v.g., certificación forestal) y la aplicación adecuada de la compensación por transformar usos del suelo hacia plantaciones de monocultivo exótico.

Estos resultados ponen en relieve la necesidad de evaluar los compromisos asociadas con la conversión a plantaciones. Por un lado, se debe considerar el valor social de los servicios ecosistémicos proporcionados por los bosques nativos y otros tipos de cobertura del suelo que están siendo reemplazados. Por otra parte, se debe monitorear a los propietarios privados que participan en el establecimiento de plantaciones para evitar o reducir los impactos ambientales negativos, lo cual es esencial en un manejo integrado del paisaje.

\section{AGRADECIMIENTOS}

Este trabajo fue patrocinado por los proyectos FONDECYT N ${ }^{\circ} 1151187$ e IAI CRN3 095 (con la ayuda de una subvención del Instituto Interamericano por el Cambio Global, con apoyo de la Fundación Nacional de Ciencias de Estados Unidos, Proyecto GEO- 1128040).

Los autores agradecen a Antonio Lara, Alejandra Stehr, Carlos Oyarzún, Dante Pinochet, Juan Schlatter, Susana Valle, Víctor Gerding y Víctor Sandoval por sus valiosos aportes al desarrollo metodológico de este trabajo.

\section{REFERENCIAS}

Ahmad I, V Verma, MK Verma. 2015. Application of Curve Number Method for Estimation of Runoff Potential in GIS environment. 2nd International Conference on Geological and Civil Engineering. IPCBEE 80(4): 16-20.

Boughton WC. 1989. A review of the USDA SCS curve number method. Australian Journal of Soil Research 27(3): 511-523.
Calder I, P Rosier, K Prasanna, S Parameswarappa. 1997. Eucalyptus water use greater than rainfall input- a possible explanation from southern India. Hydrology \& Earth System Sciences 1: 249-256.

CIREN (Centro de Información de Recursos Naturales, CL). 1999. Actualización de la información de suelos de la X Región (1999-2001). Consultado 10 nov. 2016. Disponible en http://bibliotecadigital.ciren.cl/handle/123456789/12072.

CONAF (Corporación Nacional Forestal, CL). 1998. Catastro y evaluación de los recursos vegetacionales nativos de Chile para la región de los ríos escala 1:50.000. Consultado 19 oct. 2016. Disponible en http://sit.conaf.cl/

CONAF (Corporación Nacional Forestal, CL). 2013. Catastro y evaluación de los recursos vegetacionales nativos de Chile, actualización región de los ríos 2013 escala 1:50.000. Consultado 19 oct. 2016. Disponible en http://sit.conaf.cl/.

Chen L, J Wang, W Wei, B Fu, D Wu. 2010. Effects of landscape restoration on soil water storage and water use in the Loess Plateau Region, China. Forest Ecology and Management 259: 1291-1298.

DGA (Dirección General de Aguas, CL). 2016. Reporte consulta pluviometría anual comuna de Panguipulli. Consultado 10 oct. 2016. Disponible en http://snia.dga.cl/BNAConsultas/ reportes.

Dean J, J Webb, G Jacobsen, R Chisari, P Dresel. 2015. A groundwater recharge perspective on locating tree plantations within low-rainfall catchments to limit water resource losses. Hydrology and Erath System Sciences 19: 1107-1123.

Ebrahimian M, A Ainuddin, M Soom, A Sood, L Ju Neng. 2012. Runoff estimation in steep slope watershed with standard and slope-adjusted curve number methods. Polish Journal of Environmental Studies 21(5): 1191-1202.

FAO (Organización de las Naciones Unidas para la Alimentación y la Agricultura, IT). 2015. Evaluación de los recursos forestales mundiales. Consultado 10 nov. 2016. Disponible en http://www.fao.org/3/a-i4808s.pdf

Ferreira A, C Coelho, R Walsh, R Shakesby, A Ceballos, S Doerr. 2000. Hydrological implications of soil water-repellency in Eucalyptus globulus forests, north-central Portugal. Journal of Hydrology 231-232: 165-177.

Gajardo R. 1994. La vegetación natural de Chile. Clasificación y distribución geográfica. Santiago, Chile. Editorial Universitaria. $165 \mathrm{p}$.

Grizzetti B, D Lanzanova, C Liquete, A Reynaud, A Cardoso. 2016. Assessing water ecosystem services for water resource management. Environmental Science \& Policy 61: 194203.

Heilmayr R, C Echeverría, R Fuentes, E Lambin. 2016. A plantation-dominated forest transition in Chile. Applied Geography 75: 71-82.

Huber A, A Iroumé, J Bathurst. 2008. Effect of Pinus radiata plantations on water balance in Chile. Hydrological Processes 22: 142-148.

Jaramillo A. 2014. Modelación del servicio ecosistémico de provisión y regulación hídrica bajo diferentes escenarios de uso de suelo: caso de estudio en el sur de Chile. Tesis Magister en Ciencias, Valdivia, Chile. Universidad Austral de Chile, Facultad de Ciencias. 19 p.

Kuczera G. 1987. Prediction of water yield reductions following a bushfire in ash-mixed species eucalypt forest. Journal of Hydrology 94: 215-236. 
Lara A, C Little, R Urrutia, J McPhee, C Alvarez-Garretón, C Oyarzún, D Soto, P Donoso, L Nahuelhual, M Pino, I Arisemendi. 2009. Assessment of ecosystem services as an opportunity for the conservation and management of native forests in Chile. Forest Ecology and Management 259(4): 415-424.

Laterra P, P Barral, A Carmona, L Nahuelhual. 2015. ECOSER: protocolo colaborativo de evaluación y mapeo de servicios ecosistémicos y vulnerabilidad socio-ecológica para el ordenamiento territorial. Consultado 18 jun. 2017. Disponible en http://eco-ser.com.ar/.

Li R, M Dong, J Cui, L Zhang, Q Cui, W He. 2007. Quantification of the impact of land-use changes on ecosystem services: a case study in Pingbian County, China. Environmental Monitoring and Assessment 128(1): 503-510.

Licata J, J Gyenge, M Fernández, T Schlichter, B Bond. 2008. Increased water use by ponderosa pine plantations in northwestern Patagonia, Argentina compared with native forest vegetation. Forest Ecology and Management 255: 753-764.

Little C, A Lara, J McPhee, R Urrutia. 2009. Revealing the impact of forest exotic plantations on water yield in large scale watersheds in South-Central Chile. Journal of Hydrology 374: $162-170$.

Little C, A Lara. 2010. Restauración ecológica para aumentar la provisión de agua como un servicio ecosistémico en cuencas forestales del centro-sur de Chile. Bosque 31(3): 175-178

López-Moreno J,I Zabalza, S Vicente-Serrano, J Revuelto, M Gilaberte, C Azorin-Molina, E Morán-Tejeda, J García-Ruiz, C Tague. 2014. Impact of climate and land use change on water availability and reservoir management: Scenarios in the Upper Aragón River, Spanish Pyrenees. Science of the Total Environment 493: 1222-1231.

Luzio W, O Seguel, M Casanova. 2009. Suelos de la Zona Mediterránea Húmeda (Desde $37^{\circ} \mathrm{LS}$ hasta $43^{\circ} \mathrm{LS}$ ). In Luzio W ed. Suelos de Chile. Santiago, Chile. Universidad de Chile. p. 197-239.

METEOCHILE (Dirección Meteorológica de Chile, CL). 2001. Anuario climatológico 2001. Consultado 18 jul. 2016. Disponible en http://164.77.222.61/climatologia/php/menuAnuarios.php.

Nahuelhual L, A Carmona, A Lara, C Echeverría, M González. 2012. Land-cover change to forest plantations: proximate causes and implications for the landscape in south-central Chile. Landscape and Urban Planning 107(1): 12-20.

Oyarzún C, C Frêne, G Lacrampe, A Huber, P Hervé. 2011. Propiedades hidrológicas del suelo y exportación de sedimentos en dos microcuencas de la Cordillera de la Costa en el sur de Chile con diferente cobertura vegetal. Bosque 32(1): 10-19.

Romero F, M Cozano, R Gangas, P Naulin. 2014. Zonas ribereñas: protección, restauración y contexto legal en Chile. Bosque 35(1): 3-12.

Saad R, T Koellner, M Margni. 2013. Land use impacts on freshwater regulation, erosion regulation, and water purification: a spatial approach for a global scale level. The International Journal of Life Cycle Assessment 18: 1253-1264.

USDA (United States Department of Agriculture, USA). 1989. Runoff curve number computations. Consultado 10 jul. 2016. Disponible en http://www.wcc.nrcs.usda.gov/ftpref/ wntsc/H\&H/training/runoff-curve-numbers 1.pdf

Recibido: $12 / 10 / 17$

Aceptado: 15/05/18 
